\title{
在质心系波函数具有近似的 $\mathrm{SU}_{8}$ 对称的强子结构模型
}

\author{
柯分
}

(中国科学院高能物理研究所)

\section{一、引 言}

最近实验 ${ }^{[1]}$ 观察到两个新粒子 $J$ 和 $J^{\prime}$, 它 们显著的特点是质量很大, 而寿命却很长, 这 是以往的强作用粒子所没有的性质. 对这种 现象的一种可能的解释是引入新的量子数. 在文 ${ }^{[2]}$ 中为了在弱电统一理论中避免 $\Delta S=1$ 的中性流的出现，引入了第四种“夸克”,它带 有一种新的量子数, 我们称它为 C (Charm) 荷, 并记为 C. 从结构的观点看, 基础粒子就 有四种,自然需要引人 $\mathrm{SU}_{4}$ 对称,由于这些基 础粒子的自旋是 $1 / 2$,因而与引入 $\mathrm{SU}_{6}$ 对称类 似的物理原因, 在质心系引入 $\mathrm{SU}_{8}$ 对称. 从 结构模型 ${ }^{[3]}$ 的观点看, 可以认为第四种基础 粒子也是很重的, 超强作用具有 $\mathrm{SU}_{4}$ 变换下 的不变性,在基础粒子运动速度很低时,超强 作用具有近似的 $\mathrm{SU}_{8}$ 对称. 超强作用的剩余 的相互作用，即目前所称的强作 用破坏 $\mathrm{SU}_{4}$ 和 $\mathrm{SU}_{8}$ 对称. 按照结构模型 ${ }^{[3]}$ 的观点，我们 仍然假定介子由一对正反基础粒子构成，重 子由三个基础粒子构成. 本文将从具有上述 物理特性的结构模型出发, 对粒子分类、质量 谱、粒子 $J$ 和 $J^{\prime}$ 的性质等问题进行讨论.

本文在(二)中讨论在质心系具有 $\mathrm{SU}_{8}$ 对 称的介子和重子波函数; 在 (三) 中讨论介子 和重子的质量谱; 在(四)中讨论 $J$ 和 $J^{\prime}$ 粒子 的寿命; 在 (五) 中对带 C 荷介子的弱作用湮 灭和电磁跃迁过程进行了讨论. 最后作一个 简单的小结.

\section{二、介子和重子的波函数}

在讨论波函数之前, 首先引人一种 $C$ 旋
的概念. 在 $\mathrm{SU}_{4}$ 的无穷小算符中, 存在下面 两组算符, 一组是同位旋的无穷小算符

$$
\begin{gathered}
T_{+}=\lambda_{1}^{2}=\left(\begin{array}{llll}
0 & 1 & 0 & 0 \\
0 & 0 & 0 & 0 \\
0 & 0 & 0 & 0 \\
0 & 0 & 0 & 0
\end{array}\right), \\
T_{-}=\lambda_{2}^{1}=\left(\begin{array}{llll}
0 & 0 & 0 & 0 \\
1 & 0 & 0 & 0 \\
0 & 0 & 0 & 0 \\
0 & 0 & 0 & 0
\end{array}\right), \\
T_{3}=\frac{1}{2}\left(\begin{array}{rrrr}
1 & 0 & 0 & 0 \\
0 & -1 & 0 & 0 \\
0 & 0 & 0 & 0 \\
0 & 0 & 0 & 0
\end{array}\right) .
\end{gathered}
$$

另一组是

$$
\begin{aligned}
& C_{+}=\lambda_{4}^{3}=\left(\begin{array}{llll}
0 & 0 & 0 & 0 \\
0 & 0 & 0 & 0 \\
0 & 0 & 0 & 0 \\
0 & 0 & 1 & 0
\end{array}\right), \\
& C_{-}=\lambda_{3}^{4}=\left(\begin{array}{llll}
0 & 0 & 0 & 0 \\
0 & 0 & 0 & 0 \\
0 & 0 & 0 & 1 \\
0 & 0 & 0 & 0
\end{array}\right), \\
& C_{3}=\frac{1}{2}\left(\begin{array}{rrrr}
0 & 0 & 0 & 0 \\
0 & 0 & 0 & 0 \\
0 & 0 & -1 & 0 \\
0 & 0 & 0 & 1
\end{array}\right) .
\end{aligned}
$$

显然, (2.2) 式给出的无穷小算符构成一个

1974 年 12 月 26 日收到。 
$\mathrm{SU}_{2}$ 代数, 我们称它们为 $C$ 旋. 强作用是破 坏 $C$ 旋守恒的. 在四种基础粒子中, 只有第 四种层子的 $C$ 量子数是 +1 , 其它三种层子 的 $C$ 量子数都是零, 第四种层子的奇异数是 -1 , 它的同位旋是零. 由盖尔曼一西岛规则

$$
Q=T_{3}+\frac{Y}{2}+C
$$

知道,第四种基础粒子的电荷是 $2 / 3$.从(2.2) 式可以看到

$$
C_{3}=C+\frac{s}{2}
$$

这样盖尔曼一西岛规则可以写为

$$
Q=T_{3}+C_{3}+\frac{N}{2}
$$

$N$ 是重子数. $C_{3}$ 在强作用和电磁作用中是 守恒的.

现在讨论介子和重子的波函数. 介子是 由一对正反基础粒子组成, 填充 $\mathrm{SU}_{4}$ 的 15 维 和 1 维表示, 填充 $\mathrm{SU}_{8}$ 的 63 维和 1 维表示. 介子波函数是时空旋量波函数和 $\mathrm{SU}_{4}$ 波函数 的乘积. 在 [4] 中给出的介子波函数的时空 旋量部分是

$$
\left(\begin{array}{cccc}
\frac{\pi^{0}}{\sqrt{2}}+\frac{\eta}{\sqrt{6}}+\frac{\eta^{\prime}}{\sqrt{3}} & \pi^{+} & K^{+} & K_{c}^{0} \\
x^{-} & -\frac{\pi^{0}}{\sqrt{2}}+\frac{\eta}{\sqrt{6}}+\frac{\eta^{\prime}}{\sqrt{3}} & K^{0} & K_{c}^{-} \\
K^{-} & \bar{K}^{0} & -\frac{2 \eta}{\sqrt{6}}+\frac{\eta^{\prime}}{\sqrt{3}} & \zeta^{-} \\
\bar{K}_{c}^{0} & K_{c}^{+} & \zeta^{+} & \eta^{\prime \prime}
\end{array}\right)
$$

$1^{-}$介子的 $\mathrm{SU}_{4}$ 波函数取为

$$
\left(\begin{array}{cccc}
\frac{\rho^{0}}{\sqrt{2}}+\frac{\omega}{\sqrt{2}} & \rho^{+} & K^{+*} & K_{c}^{0 *} \\
\rho^{-} & -\frac{\rho^{0}}{\sqrt{2}}+\frac{\omega}{\sqrt{2}} & K^{0 *} & K_{c}^{-*} \\
K^{-*} & \bar{K}^{0 *} & -\phi & \zeta^{-*} \\
\bar{K}_{c}^{0 *} & K_{c}^{+*} & \zeta^{+*} & J
\end{array}\right)
$$

对于 $\eta 、 \eta^{\prime} 、 \eta^{\prime \prime}$ 及 $\omega 、 \phi 、 J$ 之间的混合在下一 节中讨论. 这里的 $J$ 粒子就是最近实验 [2]

$$
\begin{aligned}
& \left.0^{-} 介\right\} \\
& \chi\left(x_{1}, x_{2}\right)=\frac{1}{2 \sqrt{2}}\left\{f_{1}\left(x_{1}, x_{2}\right)\right. \\
& \left.\quad-\frac{i}{m} \hat{p} f_{2}\left(x_{1}, x_{2}\right)\right\} \gamma_{5} \sqrt{\frac{m}{E}} \\
& \bar{\chi}\left(x_{1}, x_{2}\right)=\frac{1}{2 \sqrt{2}}\left\{f_{1}\left(-x_{2},-x_{1}\right)\right. \\
& \left.\quad+\frac{i}{m} \hat{p} f_{2}\left(-x_{2},-x_{1}\right)\right\} \gamma_{5} \sqrt{\frac{m}{E}}
\end{aligned}
$$

1-介子

$$
\begin{aligned}
& \chi_{\lambda}\left(x_{1}, x_{2}\right)=\frac{1}{2 \sqrt{2}}\left\{f_{2}\left(x_{1}, x_{2}\right)\right. \\
& \left.-\frac{i}{m} \hat{p} f_{1}\left(x_{1}, x_{2}\right)\right\} \hat{e}^{\lambda}(p) \sqrt{\frac{m}{E}} \\
& \bar{\chi}_{\lambda}\left(x_{1}, x_{2}\right)=\frac{1}{2 \sqrt{2}}\left\{f_{2}\left(-x_{2},-x_{1}\right)\right. \\
& \left.+\frac{i}{m} \hat{p} f_{1}\left(-x_{2},-x_{1}\right)\right\} \hat{e}^{\lambda+}(p) \sqrt{\frac{m}{E}}
\end{aligned}
$$

我们要求新模型保留原来 $\mathrm{SU}_{3}$ 对称的好的结 果. 对于没有第四个基础粒子的介子的波函 数取 $\mathrm{SU}_{3}$ 对称的形式. $0^{-}$介子的 $\mathrm{SU}_{4}$ 波函 数可以写成下面的形式 
$J^{\prime}$, 我们认为它是 $J$ 粒子的径向激发, 这种径 向激发态还有一个是 $\rho^{\prime}(1600)$. 利用 [5] 中 的结果可以写出具有高轨道角动量的介子波 函数,这里就不做讨论了.

重子是由三个基础粒子构成的,由于

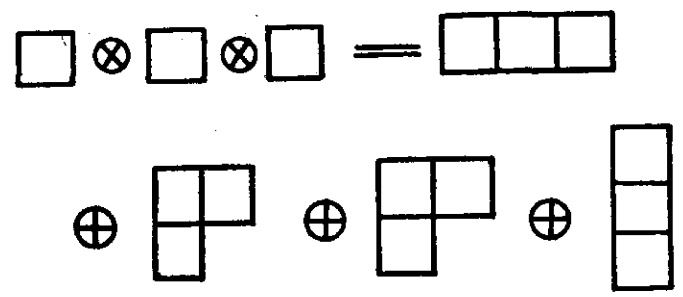

图 1

重子可以填充全对称的 20 维表示、混合对称 的 20 维不可约表示和 4 维不可约表示. 按 照 $\mathrm{SU}_{8}$ 对称, 基态的重子填充 $\mathrm{SU}_{8}$ 的 120 维 表示, 这个表示按 $\mathrm{SU}_{4} \otimes \mathrm{SU}_{2}$ 群的不可约表示 的分介为

$$
120=(20,4)+(20,2)
$$

填充全对称 20 维表示的 $3^{+} / 2$ 重子的波函数 可写为 $\chi_{\{i j k\}}\left(i, j, k\right.$ 全对称), 20 个 $3^{+} / 2$ 重 子的 $\mathrm{SU}_{4}$ 波函数分别为

$$
\begin{aligned}
\Delta^{++} & =\chi_{\{111\}}, \Delta^{+}=\sqrt{3} \chi_{\{112\}}, \\
\Delta^{0} & =\sqrt{3} \chi_{\{122\}}, \Delta^{-}=\chi_{\{222\}}, \\
\Sigma^{*+} & =\sqrt{3} \chi_{\{113\}}, \Sigma^{* 0}=\sqrt{6} \chi_{\{123\}}, \\
\Sigma^{*-} & =\sqrt{3} \chi_{\{223\}}, \Xi^{0 *}=\sqrt{3} \chi_{\{133\}}, \\
\Xi^{-*} & =\sqrt{3} \chi_{\{332\}}, Q^{-}=\chi_{\{333\}}, \\
\Sigma_{c}^{++*} & =\sqrt{3} \chi_{\{114\}}, \Sigma_{c}^{+*}=\sqrt{6} \chi_{\{124\}}, \\
\Sigma_{c}^{* *} & =\sqrt{3} \chi_{\{224\}}, \Xi_{c}^{++*}=\sqrt{3} \chi_{\{144\}}, \\
\Xi_{c}^{+*} & =\sqrt{3} \chi_{\{244\}}, \Xi_{c}^{+*}=\sqrt{6} \chi_{\{134\}}, \\
\Xi_{c}^{0 *} & =\sqrt{6} \chi_{\{234\}}, Q_{c}^{0 *}=\sqrt{3} \chi_{\{334\}}, \\
Q_{c}^{+*} & =\sqrt{3} \chi_{\{344\}}, \Omega_{c}^{++}=\chi_{\{444\}}
\end{aligned}
$$

$1^{+} / 2$ 重子填充 $\mathrm{SU}_{4}$ 的混合对称的 20 维 不可约表示, 引入四阶完全反对称的不变张 量

$$
\varepsilon_{i j k l}
$$

可以将一对反对称的下指标上升为一对反对 称的上指标. 利用这一点, $1^{+} / 2$ 重子的 $\mathrm{SU}_{4}$ 波函数可以写成

$$
N_{l}^{[m, n]}
$$

上下指标的迹等于零. 20 个 $1^{+} / 2$ 重子的 $\mathrm{SU}_{4}$ 波函数分别为

$$
\begin{aligned}
& P=N_{1}^{[3,4]}, n=N_{2}^{[3,4]}, \Sigma^{+}=N_{1}^{[2,4]}, \\
& \Sigma^{0}=\frac{1}{\sqrt{2}}\left\{N_{1}^{[1,4]}-N_{2}^{[2,4]}\right\}, \\
& \Sigma^{-}=N_{2}^{[1,4]}, \Xi^{0}=N_{3}^{[2,4]}, \Xi^{-}=N_{3}^{[1,4]}, \\
& \Lambda=\frac{1}{\sqrt{6}}\left\{N_{1}^{[1,4]}+N_{2}^{[2,4]}-2 N_{3}^{[3,4]}\right\}, \\
& \Sigma_{c}^{+}=N_{1}^{[2,3]}, \Sigma_{c}^{+}=\frac{1}{\sqrt{2}}\left\{N_{1}^{[1,3]}-N_{2}^{[2,3]}\right\}, \\
& \Sigma_{c}^{0}=N_{2}^{[1,3]}, \Xi_{c}^{++}=N_{4}^{[2,3]}, \\
& \Xi_{c}^{+}=N_{4}^{[1,3]}, Q_{c}^{0}=N_{3}^{[1,2]}, Q_{c}^{+}=N_{4}^{[1,2]}, \\
& \Lambda_{c}=\frac{1}{\sqrt{6}}\left\{N_{1}^{[1,3]}+N_{2}^{[2,3]}-2 N_{4}^{[3,4]}\right\}, \\
& \Xi_{c .}^{+}=\frac{1}{\sqrt{2}}\left\{N_{4}^{[2,4]}-N_{3}^{[2,3]}\right\}, \\
& \Xi_{c .}^{0}=\frac{1}{\sqrt{2}}\left\{N_{4}^{[1,4]}-N_{3}^{[1,3]}\right\}, \\
& \Xi_{c_{n}}^{+}=\frac{1}{\sqrt{6}}\left\{N_{4}^{[2,4]}+N_{3}^{[2,3]}-2 N_{1}^{[1,2]}\right\}, \\
& \Xi_{c_{0}}^{0}=\frac{1}{\sqrt{6}}\left\{N_{4}^{[1,4]}+N_{3}^{[1,3]}-2 N_{2}^{[1,2]\}} .(2.14)\right.
\end{aligned}
$$

从 (2.14) 可以得到每个重子的各种量子数, 这里就不再一一写出.我们仅指出, $\Xi_{c}^{+}, \Xi_{c}^{0}$; $\Xi_{c_{0}}^{+}, E_{c_{0}}^{0}$ 的同位旋、超荷和 $C$ 荷都是一样的, 但它们的 $C$ 旋是不同的, $\Xi_{c}^{++}, \Xi_{c_{1}}^{+}, \Xi^{0} ; \Xi_{c}^{+}$, $\Xi_{c_{1}}^{0}, \Xi^{-}$分别是 $C$ 旋为 1 的多重态, 而 $\Xi_{c_{0}}^{+}$, $\Xi_{c_{0}}^{0}$ 则是同位旋为 $1 / 2, C$ 旋为零的多重态.

在 [4]中给出了基态重子的旋量波函数， $3^{+} / 2$ 重子的波函数为

$$
\begin{aligned}
& B_{\alpha \beta \gamma}^{\frac{3}{2} \lambda}\left(x_{1}, x_{2}, x_{3}\right)_{i j k}^{l m n} \\
= & \left\langle 0\left|T\left\{\psi_{\alpha i}\left(x_{1}\right) \psi_{\beta j}\left(x_{2}\right) \psi_{\gamma k}\left(x_{3}\right)\right\}\right| B_{\frac{3}{2} \lambda}(p)_{l m n}\right\rangle \\
= & \frac{\sqrt{3}}{2} \Gamma_{\alpha \beta \gamma}^{\frac{3}{2} k}(p) d_{i j k}^{l m n} \sqrt{\frac{M}{E}}
\end{aligned}
$$

其中

$$
\begin{gathered}
\Gamma_{\alpha \beta \gamma}^{\frac{3}{2} \lambda}(p)=\left\{\left[f_{2}\left(x_{1}, x_{2}, x_{3}\right)\right.\right. \\
\left.\left.-\frac{i}{M} \hat{p} f_{1}\left(x_{1}, x_{2}, x_{3}\right)\right] \gamma_{\mu} C\right\}_{\alpha \beta} \psi_{\mu}^{\lambda}(p)_{\gamma}
\end{gathered}
$$




$$
\begin{aligned}
& +\left\{f_{1}\left(x_{1}, x_{2}, x_{3}\right)-f_{2}\left(x_{1}, x_{2}, x_{3}\right)\right\} \\
& \times\left\{\frac{i}{M} \hat{p} \gamma_{\varsigma} \gamma_{\mu} C\right\}_{\alpha \beta}\left\{\gamma_{5} \psi_{\mu}^{\lambda}\right\}_{r} \quad(2.16) \\
& 1^{+} / 2 \text { 重子的波函数为 } \\
& =\left\langle 0\left|T\left\{\phi_{\alpha i}\left(x_{1}\right) \psi_{\beta j}\left(x_{2}\right) \psi_{r k}\left(x_{3}\right)\right\}\right| B_{\frac{1}{2} \lambda}(p)_{l}^{\{m, n]}\right\rangle \\
& =\frac{1}{2 \sqrt{3}}\left\{\Gamma_{\alpha \beta, \gamma}(p)_{\lambda}\left(\varepsilon_{i j m n} \delta_{k l}+\varepsilon_{i k m n} \delta_{i l}\right)\right. \\
& \left.+\Gamma_{B \gamma, \alpha}(p)_{\lambda}\left(\varepsilon_{i k m n} \delta_{i l}+\varepsilon_{i k m n} \delta_{i l}\right)\right\} \sqrt{\frac{M}{E}} .
\end{aligned}
$$

其中

$$
\begin{aligned}
& \Gamma_{\alpha \beta, \gamma}(p)_{\lambda}=\left\{\left[f_{1}\left(x_{1}, x_{2}, x_{3}\right)\right.\right. \\
- & \left.\left.\frac{i}{M} \hat{p} f_{2}\left(x_{1}, x_{2}, x_{3}\right)\right] \gamma_{5} C\right\}_{\alpha \beta} u_{\lambda}(p)_{\gamma} \\
+ & \left\{f_{1}\left(x_{1}, x_{2}, x_{3}\right)-f_{2}\left(x_{1}, x_{2}, x_{3}\right)\right\} \\
\times & C_{\alpha \beta}\left\{\gamma_{5} u_{\lambda}(p)\right\}_{\gamma}
\end{aligned}
$$

利用[5]中的结果, 还可以给出

$$
\begin{aligned}
& \bar{B}_{a \beta \gamma}^{\frac{3}{2} \lambda}\left(x_{1}, x_{2}, x_{3}\right)_{i j k}^{l m n} \\
= & \left\langle B_{\frac{3}{2} \lambda} \lambda(p)_{l m n}\left|T\left\{\bar{\psi}_{a i}\left(x_{1}\right) \psi_{\beta}\left(x_{2}\right) \psi_{\gamma k}\left(x_{3}\right)\right\}\right| 0\right\rangle \\
= & \frac{\sqrt{3}}{2} d_{i j k}^{l m n} \sqrt{\frac{M}{E}} .
\end{aligned}
$$

其中

$$
\begin{aligned}
& \bar{\Gamma}_{\alpha \beta \gamma}^{\frac{3}{2} \lambda}(p)_{\lambda}=\left\{C \left[f_{2}\left(-x_{3},-x_{2},-x_{1}\right)\right.\right. \\
+ & \left.\left.\frac{i}{M} \hat{p} f_{1}\left(-x_{3},-x_{2},-x_{1}\right)\right] \gamma_{\mu}\right\}_{\alpha \beta} \phi_{\mu}^{\lambda}(p)_{\tau} \\
+ & \left\{f_{1}\left(-x_{3},-x_{2},-x_{1}\right)\right. \\
- & \left.f_{2}\left(-x_{3},-x_{2},-x_{1}\right)\right\} \\
& \times\left\{C \gamma_{5} \frac{i}{M} \hat{p} \gamma_{\mu}\right\}_{\alpha \beta}\left\{\bar{\psi}_{\mu}^{\lambda}(p) \gamma_{s}\right\}_{\gamma} \quad(2.20) \\
= & \left\langle B_{\frac{1}{2} \lambda}(p)_{l}^{\{m, n l}\left|T\left\{\bar{\phi}_{\alpha i}\left(x_{1}\right) \bar{\psi}_{\beta j}\left(x_{2}\right) \bar{\psi}_{\gamma k}\left(x_{3}\right)\right\}\right| 0\right\rangle \\
= & \frac{1}{2 \sqrt{3}}\left\{\bar{T}_{\alpha \beta \cdot \gamma}(p)_{\lambda}\left(\varepsilon_{i j m n} \delta_{k l}+\varepsilon_{i k m n} \delta_{i l}\right)\right. \\
& \left.+I_{\beta \gamma, \alpha}(p)_{\lambda}\left(\varepsilon_{i k m n} \delta_{i l}+\varepsilon_{i k m n} \delta_{i l}\right)\right\} \sqrt{\frac{M}{E}} .
\end{aligned}
$$

其中

$$
\begin{aligned}
& \Gamma_{\alpha \beta, \gamma}(p)_{\lambda}=\left\{C \left[f_{1}\left(x_{1}, x_{2}, x_{3}\right)\right.\right. \\
+ & \left.\left.\frac{i}{M} \hat{p} f_{2}\left(x_{1}, x_{2}, x_{3}\right)\right] \gamma_{5}\right\}_{\alpha \beta} \vec{u}_{\lambda}(p)_{\gamma} \\
+ & \left\{f_{1}\left(x_{1}, x_{2}, x_{3}\right)-f_{2}\left(x_{1}, x_{2}, x_{3}\right)\right\} \\
\times & C_{\alpha \beta}\left\{\bar{u}_{\lambda}(p) \gamma_{5}\right\}_{\gamma}
\end{aligned}
$$

在这样一个模型中, 基础粒子的电磁相 互作用哈密顿量为

$$
H_{i}(x)=-i e \bar{\psi}(x) Q \hat{A}(x) \psi(x)
$$

$Q$ 算符是 (2.5) 式给出的形式

$$
Q=\frac{1}{3}\left(\begin{array}{rrrr}
2 & 0 & 0 & 0 \\
0 & -1 & 0 & 0 \\
0 & 0 & -1 & 0 \\
0 & 0 & 0 & 2
\end{array}\right)
$$

与[1]中相同,等效的基础粒子电磁作用哈密 顿量是

$$
\begin{gathered}
H_{i}(x)=-i e \phi(x) Q \\
\times\left\{\hat{A}(x)-i \frac{\kappa}{4 M_{N}} \sigma_{\mu \nu} F_{\mu \nu}(x)\right\} \psi(x)
\end{gathered}
$$

按照[5]中的讨论 $\kappa=0.481$.

基础粒子的弱作用流可以写为

$$
\begin{aligned}
& J_{\mu}(x)=\bar{\phi}_{1}(x) \gamma_{\mu}\left(1+\gamma_{5}\right) \\
\times & \left\{\psi_{2}(x) \cos \theta+\psi_{3}(x) \sin \theta\right\} \\
+ & \bar{\psi}_{4}(x) \gamma_{\mu}\left(1+\gamma_{5}\right) \\
\times & \left\{-\psi_{2}(x) \sin \theta+\psi_{3}(x) \cos \theta\right\}(2.26)
\end{aligned}
$$
按照 [6] 中的讨论 $\theta=0.22$. 等效的基础粒 子的啹流可写为

$$
\begin{aligned}
J_{\mu}(x) & =\bar{\phi}_{1}(x)\left\{\gamma_{\mu}\left(1+\lambda \gamma_{s}\right)+\frac{\kappa}{2 M_{N}} \sigma_{\mu \nu} q_{\nu}\right\} \\
\times & \left\{\phi_{2}(x) \cos \theta+\psi_{3}(x) \sin \theta\right\} \\
+ & \bar{\phi}_{4}(x)\left\{\gamma_{\mu}\left(1+\lambda \gamma_{5}\right)+\frac{\kappa}{2 M_{N}} \sigma_{\mu \nu} q_{\nu}\right\} \\
\times & \left\{-\psi_{2}(x) \sin \theta+\psi_{3}(x) \cos \theta\right\}
\end{aligned}
$$

按照 [6] 中的讨论 $\lambda=0.7452$.

利用介子和重子的波函数及这些相互作 用,用 [1]中计算矩阵元的方法就可以讨论介 子和重子的弱作用和电磁作用过程. 不含 $\mathrm{C}$ 荷基础粒子的介子和重子的这些过程与过去 结构模型中的结果是相同的. 


\section{三、介子和重子的质量谱}

在 [7] 中通过对实验的分析引进了造成 同一 $\mathrm{SU}_{6}$ 多重态介子、重子质量分裂和强作 用衰变的强作用形式. 在结构模型 [1]中,认 为存在超强作用. 在现在的模型中, 可以认 为超强作用具有 $\mathrm{SU}_{4}$ 变换下的不变性; 在基 础粒子运动速度很低时, 具有近似的 $\mathrm{SU}_{8}$ 不 变性. 可以认为破坏 $\mathrm{SU}_{4}$ 对称和 $\mathrm{SU}_{8}$ 对称的 相互作用是一种剩余相互作用. 将破坏 $\mathrm{SU}_{3}$ 和 $\mathrm{SU}_{4}$ 的相互作用推广到 $\mathrm{SU}_{4}$ 的情况, 可以 写为

$$
\begin{aligned}
\mathscr{E}_{i}(x) & =f\left\{\bar{\psi}(x) \gamma_{5} \gamma_{\mu} \psi(x) \psi(x) \gamma_{5} \gamma_{\mu} \psi(x)\right. \\
+ & 2 \bar{\psi}(x) \gamma_{5} \psi(x) \cdot \bar{\psi}(x) \gamma_{5} \psi(x) \\
- & \left.3 \bar{\psi}_{3}(x) \psi_{3}(x) \bar{\psi}(x) \psi(x)\right\}
\end{aligned}
$$

(3.1) 中各项的比例系数是用 $K$ 介子、 $\eta^{\prime}$ 介子 作输人定出来的. $A$ 项将同一 $\mathrm{SU}_{8}$ 多重态中 不同的自旋态强子分开, $P$ 项将 $\mathrm{SU}_{8}$ 的不可 约表示与单态分开, $T_{3}^{3}$ 项将同一 $\mathrm{SU}_{4}$ 多重态 中含第三个基础粒子与不含第三个基础粒子 的强子分开.(3.1) 式与 [7]中的形式不同,这 种形式可以解决 [7] 中在计算介子质量时出 现的问题. 现在比 [7]中所讨论的基础粒子多 了一种, 它带有一种新量子数- C 荷.需要引 人一项反映这个粒子与其它粒子质量差的剩 余相互作用，这种项应与自旋无关，与 (3.1) 中的第三项类似, 可以引入如下形式的项

$$
g \bar{\psi}_{4}(x) \phi_{4}(x) \bar{\psi}(x) \psi(x)
$$

利用 [8]中的计算方法,取梯形近似,用(2.6)、 (2.7)、(2.8) 和 (2.9) 中的波函数及 (3.1)、 (3.2) 式所示的相互作用, 可以算出 (3.1)、 (3.2) 式所示的剩余相互作用的平均值, 从而 得到介子的质量公式如下:

$$
\begin{aligned}
m_{x}^{2}= & m_{0}^{2}-12 f m_{0} f_{1}^{2}(0), \\
m_{K}^{2}= & m_{0}^{2}-9 f m_{0} f_{1}^{2}(0)+3 f m_{0} f_{2}^{2}(0), \\
m_{\eta}^{2}= & m_{0}^{2}-8 f m_{0} f_{1}^{2}(0)+4 f m_{0} f_{2}^{2}(0), \\
m_{\eta^{\prime}}^{2}= & m_{0}^{2}+38 f m_{0} f_{1}^{2}(0)-22 f m_{0} f_{2}^{2}(0), \\
m_{K_{c}}^{2}= & m_{0}^{2}-12 f m_{0} f_{1}^{2}(0) \\
& \quad-g m_{0}\left\{f_{1}^{2}(0)+f_{2}^{2}(0)\right\},
\end{aligned}
$$

$$
\begin{aligned}
m_{\zeta}^{2}= & m_{0}^{2}-9 f m_{0} f_{1}^{2}(0)+3 f m_{0} f_{2}^{2}(0) \\
& -g m_{0}\left\{f_{1}^{2}(0)+f_{2}^{2}(0)\right\}, \\
m_{\eta}^{2}= & m_{0}^{2}+4 f m_{0} f_{1}^{2}(0)-8 f m_{0} f_{2}^{2}(0) \\
& -2 g m_{0}\left(f_{1}^{2}+f_{2}^{2}\right) \\
m_{\rho}^{2}= & m_{\omega}^{2}=m_{0}^{2}-4 f m_{0} f_{1}^{2}(0)+8 f m_{0} f_{2}^{2}(0), \\
m_{\phi}^{2}= & m_{0}^{2}+2 f m_{0} f_{1}^{2}(0)+14 f m_{0} f_{2}^{2}(0), \\
m_{K^{*}}^{2}= & m_{0}^{2}-f m_{0} f_{1}^{2}(0)+11 f m_{0} f_{2}^{2}(0), \\
m_{K_{c}}^{2}= & m_{0}^{2}-4 f m_{0} f_{1}^{2}(0)+8 f m_{0} f_{2}^{2}(0) \\
& -g m_{0}\left\{f_{1}^{2}(0)+f_{2}^{2}(0)\right\}, \\
m_{\zeta}^{2}= & m_{0}^{2}-f m_{0} f_{1}^{2}(0)+11 f m_{0} f_{2}^{2}(0) \\
& -g m_{0}\left\{f_{1}^{2}(0)+f_{2}^{2}(0)\right\}, \\
m_{J}^{2}= & m_{0}^{2}-4 f m_{0} f_{1}^{2}(0)+8 f m_{0} f_{2}^{2}(0) \\
& -2 g m_{0}\left\{f_{1}^{2}(0)+f_{2}^{2}(0)\right\} .
\end{aligned}
$$

从 (3.3) 式中可以得到下面一些质量关系:

$$
\begin{aligned}
& 4 m_{K}^{2}=3 m_{\eta}^{2}+m_{\pi}^{2}, \\
& m_{\rho}^{2}-m_{\pi}^{2}=m_{K^{*}}^{2}-m_{K}^{2}, \\
& 2 m_{K^{*}}^{2}=m_{\Phi}^{2}+m_{\rho}^{2}, \\
& m_{\rho}^{2}=m_{\omega \cdot}^{2} \\
& m_{\zeta}^{2}-m_{K_{c}}^{2}=m_{K}^{2}-m_{\pi}^{2}, \\
& 2 m_{\eta}^{2}=m_{\rho}^{2}+m_{\pi}^{2}, \\
& 24 m_{\eta^{\prime \prime}}^{2}=8 m_{\eta}^{2}+18 m_{\pi}^{2} \\
& \quad+48\left(m_{\zeta}^{2}-m_{K}^{2}\right)-2 m_{\rho}^{2}, \\
& m_{K_{c}^{2}}^{2}-m_{\rho}^{2}=m_{K_{c}}^{2}-m_{\pi}^{2}=m_{\zeta}^{2}-m_{K}^{2}, \\
& 2 m_{K_{c}^{*}}^{2}=m_{J}^{2}+m_{\rho,}^{2} .
\end{aligned}
$$

这些质量关系除第 7 条外,都与 (3.1)、(3.2) 中各项系数的具体数值无关. (3.4) 式所给 出的质量关系, 是 $\mathrm{SU}_{6}$ 对称破坏所给出的. 目 前实验给出

$$
m_{J}^{2}=9.641 \mathrm{GeV}^{2} \text {, }
$$

利用 (3.5) 中的质量关系可以将其它含 C 荷 基础粒子的介子的质量定出来, 它们为:

$$
\begin{aligned}
& m_{K_{c}^{*}}=2.265 \mathrm{GeV}, \\
& m_{\zeta^{*}}=2.303 \mathrm{GeV}, \\
& m_{K_{c}}=2.129 \mathrm{GeV}, \\
& m_{\zeta}=2.182 \mathrm{GeV},
\end{aligned}
$$

若取 $E(1420)$ 为 $\eta^{\prime}$ 介子, 则

$$
m_{\eta^{\prime \prime}}=3.109 \mathrm{GeV} \text {, }
$$

若取 $X^{\circ}(958)$ 为 $\eta^{\prime}$ 介子, 则

$$
m_{\eta^{\prime \prime}}=3.049 \mathrm{GeV} \text {. }
$$


利用 $\eta 、 \eta^{\prime}$ 和 $\eta^{\prime \prime}$ 的 $\mathrm{SU}_{4}$ 波函数及剩余相 互作用 (3.1)、(3.2) 可以得到

$$
\begin{aligned}
& \left\langle\eta^{\prime}\left|m^{2}\right| \eta\right\rangle=-2 \sqrt{2} f m_{0}\left\{f_{1}^{2}(0)+f_{2}^{2}(0)\right\}, \\
& \left\langle\eta^{\prime}\left|m^{2}\right| \eta^{\prime \prime}\right\rangle=8 \sqrt{3} f m_{0}\left\{2 f_{1}^{2}(0)-f_{2}^{2}(0)\right\},
\end{aligned}
$$

因而 $\eta^{\prime}$ 与 $\eta, \eta^{\prime}$ 与 $\eta^{\prime \prime}$ 之间有混合. 正确的 方法是将这三个介子一块混合，从而得到物 理介子的波函数. 作为一个零级近似, 分别 考虑 $\eta$ 和 $\eta^{\prime}$ 的混合及 $\eta^{\prime}$ 和 $\eta^{\prime \prime}$ 的混合, 得到 三个介子的近似的 $\mathrm{SU}_{4}$ 波函数为:

$$
\begin{aligned}
\eta_{p}= & \cos \theta_{1} \eta+\sin \theta_{1} \eta^{\prime}, \\
\eta_{p}^{\prime}= & -\sin \theta_{1} \cos \theta_{2} \eta \\
& +\cos \theta_{1} \cos \theta_{2} \eta^{\prime}-\sin \theta_{2} \eta^{\prime \prime}, \\
\eta_{p}^{\prime \prime}= & \sin \theta_{2} \eta^{\prime}+\cos \theta_{2} \eta^{\prime \prime} .
\end{aligned}
$$

其中 $\eta_{p} 、 \eta_{p}^{\prime} 、 \eta_{p}^{\prime \prime}$ 为物理的介子波函数. 其中

$$
\theta_{1}=7^{\circ} 1^{\prime}, \theta_{2}=7^{\circ} 38^{\prime} \text {, }
$$

这是用 $E(1420)$ 的质量作为输入得到的, 由 此定出

$$
f_{1}^{2}(0)=2.08 f_{2}^{2}(0)
$$

混合以后,定出

利用 (3.3) 式可以定出

$$
m_{\eta^{\prime \prime}}=3.127 \mathrm{GeV} \text {. }
$$

$$
-\frac{g}{f}=\frac{4\left(m_{J}^{2}-m_{\omega}^{2}\right)}{m_{\omega}^{2}-m_{x}^{2}}=60.8
$$

用 (3.1)、(3.2) 式的相互作用, $J$ 介子与 $\omega 、 \phi$ 介子没有混合, 因而 $\omega 、 \phi 、 J$ 介子的 $\mathrm{SU}_{4}$ 波函数就是 (2.9) 式中所给出的形式.

在这样一种分类中，实验上所发现的第 二个尖锐共振 $J^{\prime}$ 不能填充 $\mathrm{SU}_{8}$ 的基态的 $63 \oplus 1$ 维表示. 实验上已经发现了 $\rho$ 介子的 径向激发态 $\rho^{\prime}(1600)$ 介子, 可以认为 $J^{\prime}$ 介子 是 $J$ 介子的径向激发态. 在这样一个径向激 发态的 $\mathrm{SU}_{8}$ 多重态中的介子的质量公式中, $m_{0} 、 f_{1}(0) 、 f_{2}(0)$ 和基态情况是不同的. 但 是 (3.4) 式和 (3.5) 式中给出的质量关系对径 向激发态的相应的介子仍然存在. 由 $J^{\prime}$ 和 $\rho^{\prime}$ 介子的质量定出

$$
\begin{aligned}
& -2 g m_{0}^{\prime}\left\{f_{1}^{\prime 2}(0)+f_{2}^{\prime 2}(0)\right\} \\
& =m_{J^{\prime}}^{2}-m_{\rho^{\prime}}^{2}=11.1 \mathrm{GeV}^{2}
\end{aligned}
$$

利用 (3.15) 式定出

$$
f m_{0}^{\prime}\left\{f_{1}^{\prime 2}(0)+f_{2}^{\prime 2}(0)\right\}=0.0913 \mathrm{GeV}^{2} \text { (3.17) }
$$

利用这两个数值和 (3.4)、(3.5) 式给出的介 子质量关系, 可以得到其它径向激发态介子 的质量

$$
\begin{aligned}
& m_{\phi^{\prime}}=1.763 \mathrm{GeV}, m_{\omega^{\prime}}=1.6 \mathrm{GeV}, \\
& m_{\pi^{\prime}}=1.353 \mathrm{GeV}, m_{K^{\prime}}=1.45 \mathrm{GeV}, \\
& m_{\eta \gamma}=1.482 \mathrm{GeV}, m_{K_{c}^{\prime}}=2.717 \mathrm{GeV}, \\
& m_{\zeta^{\prime}}=2.767 \mathrm{GeV}, m_{K_{c}^{* \prime}}=2.848 \mathrm{GeV}, \\
& m_{\zeta^{\prime \prime}}=2.896 \mathrm{GeV}
\end{aligned}
$$

如果认为 $f_{1}^{\prime 2}(0)$ 与 $f_{2}^{\prime 2}(0)$ 的比例关系仍然是 (3.13) 式的形式, 则可得到

$$
\begin{aligned}
& m_{\eta_{r}^{\prime}}=1.983 \mathrm{GeV}, \\
& m_{\eta_{r}^{\prime \prime}}=3.684 \mathrm{GeV}
\end{aligned}
$$

用同样的方法可以得到重子的质量公 式. (3.2) 式的相互作用反映带 $\mathrm{C}$ 荷的基础 粒子与第一、二个基础粒子 (即 $p 、 n$ 基础粒 子)在强子中所引起的质量差, 从(3.15)式可 以看到, 有如下的关系

$$
\begin{aligned}
& m_{p^{\prime}}-m_{p}=20.27\left(m_{\lambda}-m_{p}\right) \\
& m_{\lambda}-m_{p}=m_{E}-m_{\Sigma}=125 \mathrm{MeV}(3.20)
\end{aligned}
$$

利用这个关系式可以得到带 $C$ 荷的 $1^{+} / 2$ 重 子的质量为

$$
\begin{aligned}
& m_{\Xi_{c_{1}}}=m_{\Xi_{c_{0}}}=3.73 \mathrm{GeV}, \\
& m_{\Sigma_{c}}=3.61 \mathrm{GeV}, m_{\Omega_{c}^{0}}=3.86 \mathrm{GeV}, \\
& m_{\Lambda_{c}}=3.53 \mathrm{GeV}, m_{\Omega_{c}^{+}}=6.14 \mathrm{GeV}, \\
& m_{\Xi_{c}}=6.01 \mathrm{GeV}
\end{aligned}
$$

(3.21) 中 $m_{\Sigma_{c}}$ 与 $m_{A_{c}}$ 的质量所以不同, 是因 为定 $m_{\Sigma_{c}}$ 时用了从 $m_{\Xi}-m_{\Sigma}$ 定出的质量. 差 $m_{\lambda}-m_{p}$, 定 $m_{A_{c}}$ 时用了从 $m_{A}-m_{N}$ 定出的 质量差 $m_{\lambda}-m_{p}$. 带 $\mathrm{C}$ 荷的 $3^{+} / 2$ 重子的质 量也可以算出来. 因为造成自旋质量差的相 互作用是 (3.1) 中的 A 型相互作用, 它是 $\mathrm{SU}_{4}$ 不变的,因而存在下面的质量关系

$$
\begin{aligned}
& m_{\Sigma_{c}^{*}}-m_{\Sigma_{c}}=m_{\Xi_{c}^{*}}-m_{\Xi_{c}} \\
= & m_{\Xi_{c_{+}}^{*}}-m_{\Xi_{c_{1}}}=m_{Q_{c}^{0}}-m_{Q_{c}^{0}} \\
= & m_{\Omega_{c}^{+*}}-m_{\Omega_{c}^{+}}=m_{\Delta}-m_{N}
\end{aligned}
$$


从 (3.22) 式得到

$$
\begin{aligned}
& m_{\Sigma_{c}^{*}}=3.91 \mathrm{GeV}, m_{\Xi_{c}^{*}}=6.31 \mathrm{GeV}, \\
& m_{\Sigma_{c}^{*}}=4.03 \mathrm{GeV}, m_{\Omega_{c}^{0}}=4.16 \mathrm{GeV}, \\
& m_{\Omega_{c}^{+}}=6.44 \mathrm{GeV}
\end{aligned}
$$

$\Omega_{c}^{++}$重子是由三个 $p^{\prime}$ 基础粒子构成的, 它的 质量是

$$
m_{2_{c}^{+}}=2 m_{\Xi_{c}}-m_{\Sigma_{c}}+m_{\Delta}-m_{N}=8.71 \mathrm{GeV}
$$

\section{四、 $J$ 和 $\boldsymbol{J}^{\prime}$ 介子的衰变}

$J$ 和 $J^{\prime}$ 介子的 $\mathrm{SU}_{4}$ 波函数都是 $\phi_{4 \text {. }}^{4}$ 由 于能量守恒的原因, 下列的强作用衰变道

$$
\begin{aligned}
J & \rightarrow \zeta+\bar{\zeta}, K_{c}+\bar{K}_{c}, \\
& \zeta^{*}+\bar{\zeta}^{*}, K_{c}^{*}+\bar{K}_{c}^{*}, \ldots \ldots \\
J^{\prime} \rightarrow \zeta^{\prime}+\bar{\zeta}^{\prime}, & K_{c}^{\prime}+\bar{K}_{c}^{\prime}, \\
& \zeta^{\prime *}+\zeta^{\prime *}, K_{c}^{\prime *}+\bar{K}_{c}^{\prime *} \ldots \ldots
\end{aligned}
$$

是禁戒的. 由于 $J$ 介子是由正反 $p^{\prime}$ 基础粒子 组成的矢量介子, 它不可能通过 (3.1)、(3.2) 式的强作用衰变到目前实验所观测到的不含 第四个基础粒子的强作用粒子. 类似的理 由, $\phi$ 介子也不能通过 (3.1) 式相互作用衰变 到 $3 \pi$, 可是实验上给出

$$
\frac{\Gamma_{\phi \rightarrow 3 \pi}}{\Gamma}=15.8 \%
$$

而 $\phi \rightarrow 3 \pi$ 道就是一种强衰变. 用 (3.1)、 (3.2)式相互作用给出的介子质量准确到 $5 \%$ 左右, 因而可以认为存在一种机制, 使得 $\phi$ 与 $\omega$ 有混合, 一个物理的 $\phi$ 介子的波函数可以 写为

$$
\phi=-\cos \theta \phi_{4}^{4}+\sin \theta \frac{1}{\sqrt{2}}\left(\phi_{1}^{1}+\phi_{2}^{2}\right)
$$

而 $\phi$ 介子 $\mathrm{SU}_{4}$ 波函数中的 $\left(\phi_{1}^{1}+\phi_{2}^{2}\right)$ 部分可 以通过 (3.1) 式相互作用衰变为 $3 \pi$, 因而

$$
\Gamma_{\phi \rightarrow 3 \pi}=\sin ^{2} \theta \Gamma_{\frac{1}{\sqrt{2}}\left(\phi_{1}^{1}+\phi_{2}^{2}\right) \rightarrow 3 x}
$$

可以用同样的机制对 $J$ 介子的衰变作一估 计. 从 (3.3) 式定出的 $\phi$ 介子的质量(用 $\omega$ 介
子的质量作为输入) 为

$$
m_{\phi_{n}}^{2}=1.059 \mathrm{GeV}^{2}
$$

考虑 $J$ 介子与 $\phi$ 介子的混合, 混合角为

$$
\begin{gathered}
\cos 2 \theta=\frac{2 m_{\phi_{0}}^{2}-m_{\phi}^{2}+m_{J}^{2}}{m_{\phi}^{2}-m_{J}^{2}}=0.9956 \\
\theta=2^{\circ} 42^{\prime}
\end{gathered}
$$

$J$ 介子的 $\mathrm{SU}_{4}$ 波函数为

$$
J=0.0471 \phi_{3}^{3}+0.9989 \phi_{4}^{4}
$$

这是一种电磁作用量级的混合, 混合的机制 以后将有专文讨论. 用 (4.4) 式得

$$
\Gamma_{J}=0.22 \times 10^{-2} \Gamma_{\phi_{3}^{3}}
$$

$\Gamma_{\phi_{3}^{3}}$ 是 $J$ 介子波函数中的 $\phi_{3}^{3}$ 部分通过 (3.1) 式的相互作用衰变为一般的强作用粒子的总 宽度. 由于 $J$ 介子的质量很重，因而 $\Gamma_{\phi_{3}^{3}}$ 可 能较大, 但由于系数很小, 因而 $J$ 介子宽度很 小,可以满足实验上给出的

$$
r_{l} \leqslant 1.9 \mathrm{MeV}
$$

的限制.

$J$ 介子可以通过电磁作用衰变为一对正 反电子或一对正反 $\mu$ 介子. 在海森堡表象中 电磁作用的哈密顿量为

$$
\mathscr{H}_{i}=-i e \bar{\psi}(x) \hat{A}(x) \psi(x)
$$

利用结构模型 [3] 的计算方法得

$$
\Gamma_{J \rightarrow 2 \epsilon}=\frac{64 \pi}{27} \alpha^{2} f_{2}^{2}(0) \frac{1}{m_{J}^{2}}
$$

在 [9] 中定出基态介子的零点波函数有下面 的形式

$$
f_{2}^{2}(0)=1.46 \times 10^{5}\left(1+\frac{m}{130.8 \mathrm{MeV}}\right)^{2} \mathrm{MeV}^{3}
$$

$m$ 是介子的物理质量,由 (4.8) 和 (4.9) 定出

$$
\Gamma_{J \rightarrow 2 \epsilon}=3.68 \mathrm{KeV}
$$

这与实验结果是一致的.

$J^{\prime}$ 介子存在下面强作用衰变道

$$
J^{\prime} \rightarrow J+2 \pi
$$

在引起强衰变的相互作用 (3.1) 和 (3.2) 中 只有 (3.2) 式所表示的项有贡献, 费曼图如 下: 


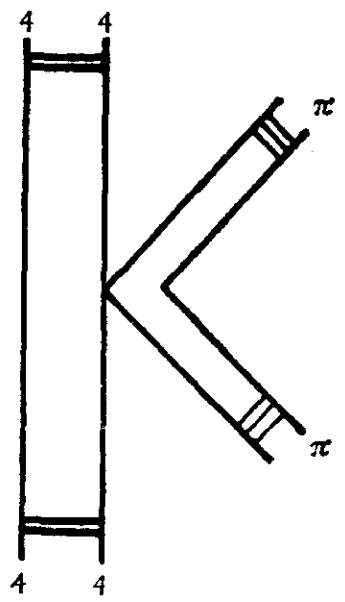

图 2

这个图的 $s$ 矩阵元为

$$
\begin{array}{r}
\left\langle 2 \pi J|s| J^{\prime}\right\rangle=-i(2 \pi)^{4} \delta\left(p_{J^{\prime}}-p-q\right) g \\
\cdot\langle 2 \pi|\bar{\psi}(0) \psi(0)| 0\rangle\left\langle J\left|\bar{\psi}_{4}(0) \psi_{4}(0)\right| J^{\prime}\right\rangle \\
q=p_{\pi^{+}}+p_{\pi^{-}}
\end{array}
$$

利用 [8] 中的计算方法 (取梯形近似，忽略 $p / M$ 级小项, $M$ 是基础粒子质量),得到

$$
\begin{aligned}
& \Gamma=\frac{g^{2}}{2(2 \pi)^{3}} \frac{m_{J} m_{x}^{2}}{m_{J^{\prime}}} \int_{-0.348}^{-0.156} d q^{2} D_{\pi}^{2}\left(q^{2}\right) D_{J}^{2}\left(q^{2}\right) \\
& \times\left(1+a^{2}+\frac{q^{2}}{2 m_{\pi}^{2}}\right)^{2}\left[\left(E_{\pi}\right)_{\text {极大 }}-\left(E_{\pi}\right)_{\text {极小 }}\right] \\
& \times\left\{3\left(a^{-2}+\frac{E}{m_{J}}\right)^{2}-\frac{p^{2}}{m_{J}^{2}}\left(1-a^{-4}\right)\right\}(4.12)
\end{aligned}
$$

其中 $p 、 E$ 分别是 $J$ 介子三维动量绝对值 和 能量, $D_{\pi}\left(q^{2}\right)$ 是两个 $\pi$ 介子的空间波函数重 叠积分, $D_{J}\left(q^{2}\right)$ 是 $J$ 与 $J^{\prime}$ 介子空间波函数的 重叠积分。

$$
\begin{aligned}
\left(E_{\pi}\right)_{\text {做大 }}= & \left\{\left[\frac{m_{J^{\prime}}-E}{2} \sqrt{1+\frac{4 m_{\pi}^{2}}{q^{2}}}\right.\right. \\
& \left.\left.+\frac{p}{2}\right]^{2}+m_{\pi}^{2}\right\}^{\frac{1}{2}} \\
\left(E_{\pi}\right)_{\text {枚小 }}= & \left\{\left[\frac{m_{J^{\prime}}-E}{2} \sqrt{1+\frac{4 m_{\pi}^{2}}{q^{2}}}\right.\right. \\
& \left.\left.-\frac{p}{2}\right]^{2}+m_{\pi}^{2}\right\}^{\frac{1}{2}}
\end{aligned}
$$

在推导 (4.12) 时用了如下关系

$$
f_{1}(x)=a f_{2}(x)
$$

$a^{2}$ 就是 (3.13) 中定出的值. 关系式 (4.13) 可 队在结构模型 [3]的基本假定下, 用取梯形近
似的 Bethe-selpeter 方程证明它, 这在以后将 有专文论述. 按照(三)中的讨论, $J^{\prime}$ 介子是 $J$ 介子的径向激发态, 如果两个介子都静止, 这两个介子的空间波函数是正交的，因而可 以认为

$$
D_{J}\left(q^{2}\right) \propto \frac{p^{2}}{m_{J}^{2}}
$$

对 $\pi$ 介子的重叠积分, 由于洛仑兹收缩效应, $\pi$ 介子运动速度愈大, $D_{n}\left(q^{2}\right)$ 就愈小, $D_{n}\left(q^{2}\right)$ 随 $q^{2}$ 增加而下降, 选取

$$
D_{\pi}\left(q^{2}\right) \propto \frac{4 m_{\pi}^{2}}{-q^{2}+4 m_{\pi}^{2}}
$$

用 (3.13) 和 (3.3) 中的质量公式定出(用 $m_{\omega}^{2}$ 、 $m_{\pi}^{2}$ 作输人),

$$
m_{0}=788 \mathrm{MeV}, f f_{2}^{2}(0)=0.0306 \mathrm{GeV},(4.16)
$$

将 $m_{0}$ 的值代人 (4.9) 式得

$$
f_{2}^{2}(0)=0.72 \times 10^{7} \mathrm{MeV}^{3},
$$

利用 (4.16)、(4.17) 和 (3.15) 式得

$$
g=2.584 \times 10^{2} \mathrm{GeV}^{2}
$$

将 (4.18)、(4.15)、(4.14) 等式代入 (4.12) 中 得

$$
\Gamma=118 \mathrm{KeV}
$$

这个结果与 $D_{\pi}\left(q^{2}\right)$ 的形式有较大的关系, 它 应该是一个随 $q^{2}$ 增加而下降的函数，(4.15) 式是 $D_{\pi}\left(q^{2}\right)$ 的一种尝试性的函数形式. 取 $D_{x}\left(q^{2}\right)=1$, 可以得 $J^{\prime}$ 强衰变宽度的上限为

$$
P<2.23 \mathrm{MeV}
$$

实验给出的值是

$$
\Gamma_{J^{\prime}} \leqslant 2.7 \mathrm{MeV}
$$

$e^{+} e^{-}$酒没实验 $\left[10\right.$ 给出 $\rho^{\prime}$ 共振峰处的截面 为 $24 \mathrm{nb}$, 由此定出

$$
\begin{aligned}
& \frac{\Gamma_{\rho^{\prime} \rightarrow c^{+}}^{+}}{\Gamma}=0.42 \times 10^{-5} \\
& \Gamma \\
& \Gamma_{\rho^{\prime} \rightarrow c^{+} e^{-}}=1.68 \mathrm{KeV}
\end{aligned}
$$

由公式

$$
\Gamma_{\rho^{\prime} \rightarrow c^{+}}-=\frac{8 \pi}{3} \alpha^{2} \frac{1}{m_{\rho^{\prime}}^{2}} f_{2}^{2}(0)_{\rho^{\prime}}
$$

定出

$$
f_{2}^{2}(0)_{\rho^{\prime}}=0.964 \times 10^{7} \mathrm{MeV}^{3}
$$

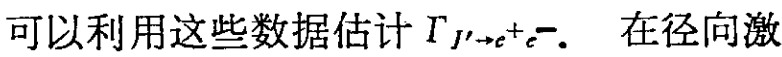


发态中可以认为 (4.9) 所表示的函数形式是 有效的,参数可能要变, 由于 $m_{\rho^{\prime}, m_{J}^{\prime}}^{2}$ 比较大, 因而可以认为

$$
\frac{f_{2}^{2}(0)_{J^{\prime}}}{f_{2}^{2}(0)_{\rho^{\prime}}}=\frac{m_{J^{\prime}}^{2}}{m_{\rho^{\prime}}^{2}}
$$

由此定出

$$
f_{2}^{2}(0)_{J^{\prime}}=5.14 \times 10^{7} \mathrm{MeV}^{3}
$$

$J^{\prime} \rightarrow e^{+} e^{-}$的衰变宽度为

$$
\Gamma_{J^{\prime} \rightarrow e^{+} e^{+}}=\frac{64 \pi}{27} \alpha^{2} \frac{1}{m_{J^{\prime}}^{2}} \cdot f_{2}^{2}(0)_{J^{\prime}}=1.5 \mathrm{KeV}
$$

$e^{+} e^{-}$湮没实验 [2] 给出在 $J^{\prime}$ 共振峰的截面 为

由此定出

$$
\sigma \geqslant 800 \mathrm{nb}
$$

$$
B=\frac{\Gamma_{J^{\prime} e^{+} e^{-}}}{\Gamma_{B}} \geqslant 0.75 \times 10^{-3}
$$

由 (4.19) 和 (4.27) 得到

$$
B_{\text {耧 }}=1.27 \times 10^{-2}
$$

这个分枝比的下限是 $0.67 \times 10^{-3}$, 与实验是 符合的.

\section{五、带 C 荷强子性质的初步讨论}

利用 (二) 中的介子、重子的波函数和电 磁作用、弱作用及强作用的哈密顿量, 可以讨 论带 C 荷的介子、重子的强衰变及一些电磁 过程和弱作用过程.

从（三）中给出的带 C 荷介子的质量, 可

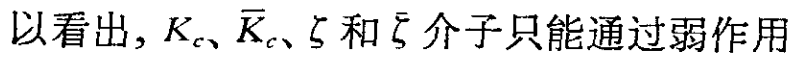
衰变, 有非轻子衰变道, 也存在如

$$
K_{c}^{-} \rightarrow \mu^{-} \bar{\nu}_{\mu}, \zeta \rightarrow \mu^{-} \bar{v}_{\mu} \cdots,
$$

的湮没过程. 利用 (2.6) 和 (2.25) 式及 [3] 中 的计算方法可以得到这两种介子的这种湮没 过程的宽度为

$$
\begin{aligned}
\Gamma_{\zeta} & =\frac{G^{2}}{2 \pi} \cos ^{2} \theta f_{2}^{2}(0)_{\zeta} m_{\mu}^{2}\left(1-\frac{m_{\mu}^{2}}{m_{\zeta}^{2}}\right)^{2}, \\
\Gamma_{K_{c}} & =\frac{G^{2}}{2 \pi} \sin ^{2} \theta f_{2}^{2}(0)_{K_{c}} m_{\mu}^{2}\left(1-\frac{m_{\mu}^{2}}{m_{K_{c}}^{2}}\right)^{2},
\end{aligned}
$$

利用 (4.9) 式给出的公式可以算出 $f_{2}^{2}(0)_{5}$ 和 $f_{2}^{2}(0)_{K_{c}}$, 将这两个值分别代人 (5.1) 和 (5.2)
中得,

$$
\begin{aligned}
\Gamma_{\zeta \rightarrow \mu \bar{v}_{\mu}} & =1.01 \times 10^{-11} \mathrm{MeV}, \\
\Gamma_{K_{c \rightarrow \mu \bar{v}_{\mu}}} & =4.82 \times 10^{-13} \mathrm{MeV} .
\end{aligned}
$$

$\eta^{\prime \prime}$ 介子可以有电磁衰变过程. 矢量介子 $K_{c}^{*}$ 、 $\bar{K}_{c}^{*} 、 \zeta^{*}$ 和 $\zeta^{*}$ 可以有电磁衰变

$$
\begin{aligned}
& K_{c}^{*} \rightarrow K_{c}+\gamma, \zeta^{*} \rightarrow \zeta+\gamma, \\
& \bar{K}_{c}^{*} \rightarrow \bar{K}_{c}+\gamma, \zeta^{*} \rightarrow \zeta+\gamma,
\end{aligned}
$$

这些介子可以有 $\mu \bar{\nu}_{\mu}$ 和 $e \bar{\nu}_{e}$ 的衰变道

$$
\begin{aligned}
& \Gamma_{\mathrm{R}_{c}^{-*} \rightarrow \mu^{-} \bar{\nu}_{\mu}}= \frac{G^{2}}{3 \pi} \sin ^{2} \theta f_{2}^{2}(0) \mathrm{K}_{c}^{*} m^{2} \mathrm{~K}_{c}^{*} \\
& \times\left(1+\frac{m_{\mu}^{2}}{2 m_{K_{c}^{2}}^{2}}\right)\left(1-\frac{m_{\mu}^{2}}{m_{K_{c}^{*}}^{2}}\right)^{2} \\
&= 1.65 \times 10^{-11} \mathrm{MeV}, \\
& \Gamma_{\zeta^{*} \rightarrow \mu^{-}-\bar{\nu}_{\mu}}=\frac{G^{2}}{3 \pi} \cos ^{2} \theta f_{2}^{2}(0)_{\zeta^{*} m_{\zeta}^{2 *}} \\
& \quad \times\left(1+\frac{m_{\mu}^{2}}{2 m_{\zeta}^{2 *}}\right)\left(1-\frac{m_{\mu}^{2}}{m_{\zeta}^{2}}\right)^{2}, \\
&= 3.59 \times 10^{-9} \mathrm{MeV} .
\end{aligned}
$$

将 (5.3) 和 (5.4) 式中 $\mu$ 介子的质量换为电 子的质量, 就得到这两种矢量介子衰变到 $e^{-} \bar{\nu}_{c}$ 的宽度. 由于 $m_{\zeta^{*}} \gg m_{\mu}, m_{K_{c}^{*}} \gg m_{\mu}$, 因 而这种矢量介子衰变到 $\mu \vec{\nu}_{\mu}$ 和 $e \bar{\nu}_{c}$ 的几率几 乎是相等的.

处在径向激发态的介子, 它们可以有强 衰变.

$1^{+} / 2$ 的带 C 荷的重子 $\Sigma_{c} 、 E_{c} \Lambda_{c}, \Omega_{c}^{0} 、 \Omega_{c}^{+}$ 主要是非轻子衰变, $\Sigma_{c}^{0}$ 可以有如下形式的电 磁衰变,

$$
\Sigma_{c}^{0} \rightarrow \Lambda_{c}+\gamma,
$$

$\Xi_{c_{1}}$ 与 $\Xi_{c_{0}}$ 之间应该有一个较小的质量差, 它 们之间质量较低的主要是非轻子衰变, 质量 较高的一个可以通过放一个 $r$ 光子到质量较 低的一个.

$3^{+} / 2$ 带 $\mathrm{C}$ 荷的重子主要是强作用衰变, 如,

$$
\Sigma_{c}^{*} \rightarrow \Sigma_{c}+\pi, \Sigma_{c}^{*} \rightarrow \Lambda_{c}+\pi, \cdots
$$

等, 这样的重子寿命很短.

带 C 荷的介子和重子可以在强作用中成 对产生, 现有的高能加速器的能量是可以 
成对产生这些粒子的, 而 $J 、 J^{\prime} 、 \eta^{\prime \prime}$ 介子则是 可以单个强作用产生的. 带 C 荷粒子也可以 在高能中微子反应中单个产生, 也可以在 $e^{+} e^{-}$湮没过程中成对产生,这可以在 $e^{+} e^{-}$湮 没的总截面上带来一种间行为, 目前 $S L A C$ 的 $e^{+} e^{-}$对碰机的能量可以成对产生 $K_{c} 、 \zeta$ 介 子。

\section{六、讨 论}

1. 通过以上的讨论可以看到, 如果认为 $J 、 J^{\prime}$ 介子象文中所讨论的那样, 填充 $\mathrm{SU}_{8}$ 多 重态，那末,这样一个模型可以解释 $J$ 和 $J^{\prime}$ 的 性质. 对于这个模型最重要的检验是看实验 能否发现理论所预言的一些介子和重子. 目 前有迹象表明这样的粒子是存在的，如实验 发现两个

$$
\nu+N \rightarrow \mu^{+} \mu^{-} \cdots
$$

的反应事例, 可以认为是 $\bar{\zeta}$ 介子产生以后又 衰变出一个 $\mu^{+}$介子. 在 [11] 中也报道了一 种新粒子, 有可能是 $K_{c} 、 \zeta$ 中的一种.

2. 在(二)中给出的重子的 $\mathrm{SU}_{8}$ 波函数是 全对称的,这里有一个统计问题,可以用三阶 的综合统计 [12], 也可以引入一个新的 $\mathrm{SU}_{3}$ 群, 认为有十二种基础粒子, 作 $\mathrm{SU}_{3} \otimes \mathrm{SU}_{8}$ 对
称的重子波函数, 目前的介子和重子都是这 个 $\mathrm{SU}_{3}$ 群的单态.

\section{参 考 资 料}

[1] Samuel, C. C. Ting, et al., Phys. Rev. Lett., 33 (1974), 1404;

Augustin, J. E., et al., Phys. Rev. Lett., 33 (1974), 1406;

Bacei, C., et al., Phys. Rev. Lett., 33 (1974), 1408.

[2] Glashow, S., et al., Phys. Rev., D2 (1970), 1285.

[3] 北京基本粒子组, 1966 年北京物理讨论会论文.

[4] 李炳安, 物理学报, 1974,1 .

[5]李炳安, 层子模型中 $1^{+} / 2$ 重子的电磁性质和 $\triangle(1236)$ 的电生光生现象, 待发表。

[6] 阮同泽、李炳安, 层子模型中 $1^{+} / 2$ 重子的半轻子 衰变和高能中微子准弹性产生, 待发表。

[7] 中国科学院原子能研究所基本柆子理 论组, 原子 能, 1966, 7-8, 460 .

[ 8 ] Mandelstam, S., Proc. Roy. Soc., 233 (1955), 248.

[9] 这是阮同泽和李炳安在讨论 $\pi \rightarrow \mu \nu$ 和 $\kappa \rightarrow \mu \nu$ 过 程时所给出的一种函数形式.

[10] Chinowsky, W., IV International Conference on Experimental Meson Spectroscopy, 1974, April, 26, 27.

[11] Kiyoshi Niu, et al., Prog. Theor. Phys., 46 (1971), 1644

[12] Green, H. S., Phys. Rev., 90 (1953), 270; Green berg, o. W., Phys. Rev. Lett., 13 (1964), 598.

\title{
在正负电子湮灭过程中噟标介子和矢量介子的产生
}

\author{
黄涛 \\ (中国科学院高能物理研究所)
}

近来，正、负电子湮灭过程的实验结果 引起人们极大的兴趣 ${ }^{[1]}$. 特别是比值 $R=$ $\sigma\left(e^{+} e^{-} \rightarrow\right.$ 强子 $) / \sigma\left(e^{+} e^{-} \rightarrow \mu^{+} \mu^{-}\right)$随能量增 加而上升的事实, 例如在质心系里能量在 $3 \mathrm{GeV}$ 时 $R$ 约为 $2-3$, 在 $5 \mathrm{GeV}$ 时 $R$ 约为 $4-7^{[1]}$, 这与许多模型认为 $R$ 趋于常 数的结 论相冲突. 实验上出现的新结果对现有理论
的冲击往往将揭示出更本质的东西*.

为了分析研究这些新的结果, 本文在层 子模型 ${ }^{[2]}$ 的基础上作了初步的探讨. 从正、负 电子湮灭的实验结果可以看出某些单个 $x$ 介

本文 1974 年 12 月 26 日收到.

* 在本工作完成以后,获悉 $J$ 粒子被发现的消息, 它是 一个矢量介子, 本文的某些讨论只要作适当的扩充 就可加以探讨. 